\title{
Comparative genomics of drug resistance in Trypanosoma brucei rhodesiense
}

\author{
Fabrice E. Graf ${ }^{1,2} \cdot$ Philipp Ludin $^{1,2} \cdot$ Christian Arquint $^{1,2} \cdot$ Remo S. Schmidt $^{1,2} \cdot$ Nadia Schaub $^{1,2}$. \\ Christina Kunz Renggli ${ }^{1,2}$ - Jane C. Munday ${ }^{3}$ - Jessica Krezdorn ${ }^{3}$ - Nicola Baker ${ }^{4,6}$ - David Horn ${ }^{4}$. \\ Oliver Balmer $^{1,2}$ - Adalgisa Caccone ${ }^{5}$ Harry P. de Koning ${ }^{3}$ Pascal Mäser ${ }^{1,2}$ (I)
}

Received: 30 June 2015/Accepted: 1 March 2016/Published online: 14 March 2016

(c) The Author(s) 2016. This article is published with open access at Springerlink.com

\begin{abstract}
Trypanosoma brucei rhodesiense is one of the causative agents of human sleeping sickness, a fatal disease that is transmitted by tsetse flies and restricted to SubSaharan Africa. Here we investigate two independent lines of $T$. $b$. rhodesiense that have been selected with the drugs melarsoprol and pentamidine over the course of 2 years, until they exhibited stable cross-resistance to an unprecedented degree. We apply comparative genomics and transcriptomics to identify the underlying mutations. Only few mutations have become fixed during selection. Three genes were affected by mutations in both lines: the aminopurine transporter $A T 1$, the aquaporin $A Q P 2$, and the RNA-binding protein $U B P 1$. The melarsoprol-selected line carried a large deletion including the adenosine transporter
\end{abstract}

F. E. Graf and P. Ludin contributed equally.

Electronic supplementary material The online version of this article (doi:10.1007/s00018-016-2173-6) contains supplementary material, which is available to authorized users.

Pascal Mäser

pascal.maeser@unibas.ch

1 Swiss Tropical and Public Health Institute, Socinstrasse 57, 4051 Basel, Switzerland

2 University of Basel, 4000 Basel, Switzerland

3 Institute of Infection, Immunity and Inflammation, University of Glasgow, Glasgow G12 8TA, UK

4 Biological Chemistry and Drug Discovery, School of Life Sciences, University of Dundee, Dow Street, Dundee DD1 5EH, UK

5 Department of Ecology and Evolutionary Biology, Yale University, New Haven, CT, USA

6 Present Address: The University of Kent, Canterbury, Kent CT2 7NZ, UK gene $A T 1$, whereas the pentamidine-selected line carried a heterozygous point mutation in AT1, G430R, which rendered the transporter non-functional. Both resistant lines had lost $A Q P 2$, and both lines carried the same point mutation, R131L, in the RNA-binding motif of UBP1. The finding that concomitant deletion of the known resistance genes $A T 1$ and $A Q P 2$ in $T$. b. brucei failed to phenocopy the high levels of resistance of the $T$. $b$. rhodesiense mutants indicated a possible role of UBPI in melarsoprolpentamidine cross-resistance. However, homozygous in situ expression of $U B P 1$-Leu ${ }^{131}$ in T. b. brucei did not affect the sensitivity to melarsoprol or pentamidine.

Keywords African trypanosomes - Aquaporin . RNA-binding protein Purine permease - Pentamidine . Melarsoprol

$\begin{array}{ll}\text { Abbreviations } \\ \text { MPXR } & \text { Melarsoprol-pentamidine cross-resistance } \\ \text { SLT } & \text { Spliced leader trapping } \\ \text { AQP2 } & \text { Aquaglyceroporin 2 } \\ \text { AT1 } & \text { Adenosine transporter 1 } \\ \text { Tet } & \text { Tetracycline } \\ \text { UBP1 } & \text { Uridine-rich binding protein 1 } \\ \text { VSG } & \text { Variant surface glycoprotein }\end{array}$

\section{Introduction}

Human African trypanosomiasis (HAT, also known as sleeping sickness) is a fatal disease caused by Trypanosoma brucei rhodesiense and T. $b$. gambiense in Eastand West-Africa, respectively. These protozoan parasites are transmitted by tsetse flies and proliferate extracellularly 
in the bloodstream and lymph of their mammalian hosts, evading the adaptive immune response through antigenic variation of their variant surface glycoprotein (VSG) coat. Ultimately the trypanosomes also infest the cerebrospinal fluid, causing the 'sleeping sickness' syndrome of infected patients. Trypanosoma brucei has an approximate haploid genome size of $35 \mathrm{Mb}$, which can vary up to $25 \%$ [1]. Excluding the kinetoplast (i.e., mitochondrial) DNA leaves a nuclear core genome of about $26 \mathrm{Mb}$, divided into 11 megabase-sized chromosomes, where the vast majority of the predicted $>9000$ protein-coding genes are located [2]. The treatment of HAT relies on just five drugs. Patients in the first, hemolymphatic stage are treated with suramin ( $T$. $b$. rhodesiense) or pentamidine (T. b. gambiense). In the second stage, when the trypanosomes have invaded the central nervous system, melarsoprol or nifurtimox-eflornithine combination therapy (NECT; only for $T$. $b$. gambiense) are used [3]. These drugs are outdated, impractical, and suffer from severe adverse effects. Melarsoprol, in particular, causes unacceptable toxicity [4]. Furthermore, melarsoprol treatment failure rates of up to $30 \%$ have been reported throughout sub-Saharan Africa [5-8]. New drugs that are safe and orally available are presently in clinical development [9]. Meanwhile, it is essential to sustain the current drugs in spite of their shortcomings, which requires an understanding of the mechanisms of drug resistance. This will also help avoid cross-resistance between current treatments and those in development.

The molecular mechanisms of drug resistance have predominantly been studied in $T$. $b$. brucei, which is nonpathogenic to humans and widely used as a model in molecular parasitology. A phenomenon that has been repeatedly observed is melarsoprol-pentamidine cross-resistance (MPXR), i.e., trypanosomes selected for resistance with a melaminophenyl arsenical turned out to be crossresistant to pentamidine and vice versa [10-12]. This phenomenon was attributed to the finding that the uptake of melarsoprol and pentamidine into the trypanosomes is mediated by the same set of transporters: the aminopurine permease P2 [13, 14], encoded by the gene $A T 1$, and a high-affinity pentamidine transport activity designated as HAPT1 [15, 16] recently shown to correspond to the aquagylceroporin AQP2 [17, 18]. Mutations in these transporters were described from drug-resistant $T$. brucei ssp. isolates from the field [19-21]. In the lab, MPXR was phenocopied by reverse genetics. Homozygous deletion of either $A T 1$ or $A Q P 2$ resulted in resistance to both melarsoprol and pentamidine. However, the obtained resistance factors were only between 2 and 3 for melarsoprol and pentamidine in ATl null trypanosomes [22, 23], respectively, and 2 for melarsoprol and 15 for pentamidine in $A Q P 2$ null mutants [17].
Here we investigate two clonal drug-resistant lines of $T$. $b$. rhodesiense that exhibit markedly higher levels of MPXR than observed after deletion of either $A T 1$ or $A Q P 2$. The lines T. $b$. rhodesiense STIB900-M and STIB900-P had been selected in vitro from their drug-susceptible parent T. b. rhodesiense STIB900 by continuous in vitro exposure to increasing concentrations of melarsoprol and pentamidine, respectively, over a period of 24 months [24]. Finally, both lines exhibited a high level of MPXR with in vitro resistance factors up to 80 (the resistance factor was defined as $\mathrm{IC}_{50}$ of the selected line divided by the $\mathrm{IC}_{50}$ of the drug-sensitive parent). This phenotype was stable in the absence of drug pressure and after passage through mice. An initial genotypic characterization demonstrated that ATl had been lost in STIB900-M but was still present in STIB900-P [24]. Evidently, given the high level of drug resistance, further mutations must be involved. We have performed whole genome sequencing and RNA-Seq of the parental T. b. rhodesiense STIB900 and its resistant derivatives STIB900-M and STIB900-P, aiming to elucidate the molecular mechanisms underlying the unprecedented level of MPXR by comparative genomics and transcriptomics.

\section{Materials and methods}

\section{T. b. rhodesiense lines}

Trypanosoma brucei rhodesiense STIB900 is a derivative of STIB704, isolated from a male patient at St. Francis Hospital in Ifakara, Tanzania, in 1981. After several passages in rodents and a cyclic passage through a tsetse fly (Glossina morsitans morsitans), a cloned population was adapted to axenic growth. T. b. rhodesiense STIB900-M and STIB900-P had been selected independently in vitro for resistance to melarsoprol and pentamidine, respectively [24]. Bloodstream-form trypanosomes were propagated in vitro as described in [20] and adapted from [25]. Cells were counted with the CASY ${ }^{\circledR}$ Cell Counter system (Roche). Large numbers of trypanosomes for DNA isolation were obtained by inoculating female Naval Medical Research Institute (NMRI) mice (Harlan Laboratories) with $10^{6}$ trypanosomes. At peak parasitemia, the trypanosomes were harvested and separated from the blood cells on DEAE-cellulose columns [26].

\section{In vitro drug sensitivity}

For all the STIB900 lines drug sensitivities were determined with the Alamar blue assay [27]. T. b. brucei B48 drug sensitivities were determined as described using a slightly modified protocol [28]. The SoftMax Pro software 
and Prism 5 (GraphPad) were used to calculate $50 \%$ inhibitory concentrations $\left(\mathrm{IC}_{50}\right)$ by non-linear regression fitting to a sigmoidal dose-response curve. All assays were performed at least three times independently, each in duplicate or triplicate. The following compounds were tested, each individually: melarsoprol (Sanofi-Aventis/ WHO), eflornithine (Sanofi-Aventis), DB75 (Immtech), fexinidazole (DNDi, Geneva), nifurtimox (WHO, Geneva), suramin (Bayer), pentamidine isethionate, diminazene aceturate, phenylarsine oxide, aminopurinol, cordycepin, adenosine arabinoside, and tubercidin (Sigma). Statistical tests were performed with GraphPad Prism 5.0.

\section{Isolation of nucleic acids}

Genomic DNA was isolated by phenol/chloroform extraction from bloodstream-form trypanosomes propagated in mice. To check for contamination with mouse DNA we performed PCR with primers for mouse glyceraldehyde-3phosphate-dehydrogenase (GAPDH) and mouse cDNA as a positive control. For each $T$. $b$. rhodesiense line, about $60 \mu \mathrm{g}$ of genomic DNA was prepared for sequencing. Total RNA was isolated from exponentially growing cultures of trypanosomes $\left(10^{6}\right.$ cells $\left./ \mathrm{ml}\right)$ with TRIzol (Life technologies). Equal amounts of total RNA were pooled from three independent isolations, and from each pooled sample $12 \mu \mathrm{g}$ were used for sequencing.

\section{Spliced leader trapping}

Library preparation and RNA-Seq were performed according to the spliced leader trapping (SLT) protocol [29]. This is a modification of the standard Illumina protocol that uses the $T$. brucei $39 \mathrm{nt}$ spliced leader sequence (which is a peculiarity of trypanosomatids and gets ligated to the $5^{\prime}$ end of every mRNA) for 2nd strand cDNA synthesis and sequencing. Two independent experiments were performed: run 1 on the Genome Analyzer IIx (Illumina) and run 2 on the HiSeq 2000 (Illumina). The Fastq files were read into the Spliced Leader ADDition (SLADD) program [29] and mapped onto the reference genome sequence of $T$. brucei TREU927 [2], using MAQ [30] with $n=3$ and with a read length of $\geq 24$. Multimapping reads were separated from single mappers by an alignment quality threshold of 30 . Read counts were normalized according to library size and expressed as tags per million reads (TPM). Statistical analysis for differentially expressed genes was performed with the DESeq package in $\mathrm{R}$ [31]. DESeq uses a negative binomial distribution and a shrinkage factor for the distribution's variance. Only mapped reads (raw counts) with a stringent quality score of $q<30$ and data from both performed SLT runs were included.

\section{Whole genome sequencing}

Whole genome sequencing of $T$. $b$. rhodesiense STIB900 was carried out on the Illumina HiSeq 2000 platform. Two times 12,243,924 paired-end reads of $76 \mathrm{~b}$ were mapped chromosome-wise to the reference genome $T$. b. brucei 927 (v5) using MIRA (v3.9.16) [32]. Gene models from the reference genome $T$. $b$. brucei 927 were transferred to the assembled STIB900 genome using rapid annotation transfer tool (RATT) [33] from the post assembly genome improvement toolkit (PAGIT) [34] package. Whole genome sequencing of $T$. $b$. rhodesiense STIB900-M and STIB900-P was carried out on the Genome Sequencer FLX Titanium by Roche/454. Two shotgun runs per line were performed. FASTQ format was extracted from .sff files using 'SFF converter' from Galaxy [35]. High-quality (HQ) reads were mapped to the assembled STIB900 genome, indexed with word length 13 and skip step 1, using the program SMALT (ftp.sanger.ac.uk/pub4/resources/ software/smalt). Consensus sequence and variants relative to the assembled STIB900 genome were identified with 'mpileup' from SAMtools [36]. Ad hoc Perl scripts were used to compare nucleotide variants between the mapped reads of STIB900-M, STIB900-P, and the assembled STIB900 genome. For comparison also STIB900 reads generated on the Roche/454 platform were mapped to the assembled STIB900 genome. SNPs were called if they had a read depth of at least five high-quality bases (DP4 $\geq 5$ ) and a read mapping quality of minimum 20 (mapq $\geq 20$ ). All identified SNPs, indels, and gene deletions were inspected manually using Artemis [37]. The Roche/454 reads are accessible via the European Nucleotide Archive (http://www.ebi.ac.uk/ena) under accession number PRJEB12780.

\section{PCR amplification and Sanger sequencing}

The PCR primers to amplify the $3.2 \mathrm{~kb} A Q P 2 / A Q P 3$ tandem locus were AQP2/3_F (aagaaggctgaaactccacttg) and AQP2/3_R (tgcactcaaaaacaggaaaaga), annealing at $58{ }^{\circ} \mathrm{C}$. AT1-G430R was amplified from genomic DNA of STIB900-P with primers AT1_F (gaaatcccegtctttctcac) and AT1_R (atgtgctgagcctttttcctt), annealing at $56{ }^{\circ} \mathrm{C}$. The PCR product was purified on silica-membrane columns (Nucleospin gel and PCR clean up, Macherey-Nagel) and digested with NruI (New England Biolabs), run on a $1.5 \%$ agarose gel and visualized with ethidium bromide. UBPl and UBP1-R131L were amplified from STIB900 and STIB900-M, respectively, with the primers UBP1_F (ccgctctagatctcaggttccactggcttc) and UBP1_R (ccgcggatcctcatttacgggcaggccgac). 


\section{Plasmid construction, transfection and knock-out generation}

The gene encoding the AT1-G430R mutant was amplified by PCR from genomic DNA of STIB900-P and the product was ligated into the expression vector pHD1336 [38] to give pHDK68. The plasmid was verified by Sanger sequencing (Source BioScience, Nottingham, UK) for the presence of the expected mutation and linearized with NotI prior to transfection into $T$. b. brucei clone B48, which lack the $A T 1$ gene and the high-affinity pentamidine transporter [16]. B48 parasites were washed in Human T Cell Solution for transfection with an Amaxa Nucleofector [39]. Transfectants were cloned by limiting dilution in standard HMI11 medium [40] containing $5 \mu \mathrm{g} / \mathrm{ml}$ blasticidin for selection of the positive transfectants. Correct integration of the expression cassettes was tested by PCR.

Trypanosoma brucei rhodesiense $2 \mathrm{~T} 1$ aqp $2 / 3^{-1-}$ cells were assembled using a I-SceI meganuclease-based geneconversion approach. Briefly, blasticidin deaminase (BSD) and neomycin-phosphotransferase (NPT) cassettes were used to replace the $A Q P 2 / 3$ locus. Meganuclease cleavage of the NPT cassette was then used to trigger replacement with, and duplication of, the $B S D$ cassette. AT1 was disrupted in the resulting $2 \mathrm{~T} 1$ aqp $2 / 3^{-/-}$cells by replacing the first allele of the 1392 bp ORF with PCR-amplified NPT containing 100 bp overhangs identical to the UTRs of AT1, followed by selection with $5 \mu \mathrm{g} / \mathrm{ml} \mathrm{G} 418$. The second allele was replaced with PAC plus 500 bp ATI UTR on either end, followed by selection with $0.1 \mu \mathrm{g} / \mathrm{ml}$ puromycin. Homozygous deletion of $A T 1$ was verified by PCR (Figure S1).

\section{Reverse genetics of UBP1}

For overexpression of UBP1, the UBP1 and $U B P 1-R 131 L$ PCR products were cloned into pRPai ${ }^{\mathrm{GFPx}}$ [41] via $\mathrm{XbaI}$ and BamHI. T. b. brucei 2T1 cells [42] were transfected with AscI-digested plasmids in Tb-BSF nucleofection buffer [43] using the Amaxa nucleofector (Lonza) with program Z-001. Transfectants were cloned by limiting dilution and selected with $2.5 \mu \mathrm{g} / \mathrm{ml}$ hygromycin. PCR and Sanger sequencing confirmed correct integration and sequence of the transgene.

To introduce the mutant UBP1-R131L in 2T1 cells in situ, a plasmid carrying the mutation and a blasticidin resistance gene $(B S D)$ in the $5^{\prime}$ UTR of $U B P 1$, used as a selection marker, was constructed (Supplementary Figure S3A). The synthetic DNA was obtained from GenScript (Piscataway Township, NJ, USA), integrated between the HindIII and BamHI sites of cloning plasmid pUC57. DNA for transfection was prepared by PCR amplification of the insert using primers BLA_UBP1mut_F1 (ttgcattcgetcetttccet) and BLA_UBP1mut_R1 (ccttcagtagtttgttgagg) and subsequent purification as described above. 2T1 cells were transfected with an Amaxa Nucleofector using program Z-001 and clones were obtained as described above. Cells were selected with 5 and $10 \mu \mathrm{g} / \mathrm{ml}$ blasticidin. Correct homozygous integration was verified by PCR (Supplementary Figure S3B) and Sanger sequencing (Fig. 6b).

\section{SDS-PAGE and western blotting}

Cells were lysed in NUPAGE ${ }^{\circledR}$ LDS sample buffer (Life Technologies) and samples were loaded on precast 4-12\% Bis-Tris Gradient Gels (NuPAGE Novex ${ }^{\circledR}$, Life Technologies) in MES running buffer and transferred to nitrocellulose membranes using the iBlot dry-blotting system (Novex $^{\circledR}$, Life Technologies). Membranes were blocked in $5 \%$ milk in PBS/Tween-20 and incubated with primary antibodies in $5 \%$ milk in TBS/Tween-20 overnight at $4{ }^{\circ} \mathrm{C}$. Membranes were washed and incubated with peroxidase-conjugated secondary antibodies in $5 \%$ milk PBS/ Tween-20 for $2 \mathrm{~h}$ at room temperature. Blots were developed using the ECL Western Blotting Substrate (Pierce) using a ChemiDoc ${ }^{\mathrm{TM}}$ MP Gel Imaging System (Biorad). Primary Antibodies used: rabbit anti-GFP (Abcam, Ab290), mouse anti-BiP (kind gift of Prof A. Schneider). Secondary Antibodies used: goat anti-rabbit (SouthernBiotech: 4050-05), polyclonal rabbit anti-mouse HRP (Dako, Baar, Switzerland).

\section{Results}

\section{Phenotypic profiling of high-level melarsoprol-} pentamidine cross-resistance

Before venturing into genomics we performed an in-depth phenotypic profiling of the parental $T$. $b$. rhodesiense STIB900 and its two drug-resistant derivatives STIB900-M and STIB900-P. Drug sensitivities were quantified as $50 \%$ inhibitory concentrations $\left(\mathrm{IC}_{50}\right)$ towards the five clinical trypanocides (pentamidine, suramin, melarsoprol, eflornithine, and nifurtimox), two clinical candidates (fexinidazole and the diamidine DB75), and selected experimental compounds (Table 1). STIB900-M and STIB900-P exhibited similar resistance profiles, namely strong MPXR with cross-resistance to other diamidines (i.e., diminazene aceturate and DB75) and adenosine analogs [cordycepin (3'-deoxyadenosine), tubercidin (7deazaadenosine), vidarabine (adenosine arabinoside)]. The melarsoprol-selected line generally had higher resistance factors than the pentamidine-selected line to the adenosine analogs and other typical AT1 substrates [23] such as DB75 
Table 1 In vitro drug sensitivity profiles

\begin{tabular}{|c|c|c|c|c|c|c|c|c|}
\hline & \multicolumn{3}{|c|}{$\mathrm{IC}_{50} \pm$ standard deviation $[\mathrm{nM}]$} & \multicolumn{3}{|l|}{$p$ value } & \multicolumn{2}{|c|}{ Resistance factor } \\
\hline & STIB900 & STIB900-P & STIB900-M & $\begin{array}{l}\text { STIB900 vs. } \\
\text { 900-P }\end{array}$ & $\begin{array}{l}\text { STIB900 vs. } \\
\text { 900-M }\end{array}$ & $\begin{array}{l}\text { STIB900-P vs. } \\
\text { 900-M }\end{array}$ & $\begin{array}{l}\text { STIB900-P to } \\
900\end{array}$ & $\begin{array}{l}\text { STIB900-M to } \\
900\end{array}$ \\
\hline Melarsoprol & $6.0 \pm 3.4$ & $84 \pm 52$ & $170 \pm 63$ & 0.040 & $<0.0001$ & 0.026 & 14 & 28 \\
\hline Pentamidine & $2.8 \pm 0.8$ & $130 \pm 69$ & $210 \pm 93$ & 0.033 & 0.0008 & 0.17 & 47 & 76 \\
\hline Diminazene & $3.8 \pm 1.5$ & $18 \pm 5.4$ & $25 \pm 10$ & 0.012 & 0.0003 & 0.25 & 4.7 & 6.5 \\
\hline DB75 & $3.7 \pm 0.9$ & $22 \pm 8$ & $64 \pm 17$ & 0.046 & $<0.0001$ & $<0.0001$ & 5.9 & 17 \\
\hline Suramin & $135 \pm 62$ & $76 \pm 32$ & $125 \pm 25$ & 0.11 & 0.92 & 0.21 & 0.6 & 0.9 \\
\hline Nifurtimox & $1100 \pm 550$ & $1100 \pm 460$ & $1500 \pm 620$ & 0.99 & 0.51 & 0.55 & 1.0 & 1.4 \\
\hline Fexinidazole & $3200 \pm 1100$ & $2500 \pm 980$ & $6100 \pm 2500$ & 0.80 & 0.041 & 0.013 & 0.8 & 1.9 \\
\hline Eflornithine & $5200 \pm 1400$ & $1300 \pm 640$ & $2700 \pm 480$ & $<0.0001$ & 0.0033 & 0.094 & 0.3 & 0.5 \\
\hline Cordycepin & $0.46 \pm 0.1$ & $2.2 \pm 0.54$ & $7.0 \pm 1.5$ & 0.026 & $<0.0001$ & $<0.0001$ & 4.7 & 15 \\
\hline Vidarabine & $265 \pm 108$ & $400 \pm 52$ & $650 \pm 190$ & 0.24 & 0.0006 & 0.0186 & 1.5 & 2.5 \\
\hline Tubercidin & $26 \pm 6$ & $100 \pm 19$ & $4300 \pm 1000$ & 0.97 & $<0.0001$ & $<0.0001$ & 3.8 & 163 \\
\hline Aminopurinol & $1500 \pm 190$ & $5400 \pm 1100$ & $2000 \pm 630$ & $<0.0001$ & 0.58 & $<0.0001$ & 3.6 & 1.3 \\
\hline Phenylarsine & $0.52 \pm 0.13$ & $0.65 \pm 0.11$ & $0.75 \pm 0.08$ & 0.26 & 0.028 & 0.45 & 1.3 & 1.4 \\
\hline
\end{tabular}

Mean $\mathrm{IC}_{50}$ values, $p$ values of Anova one-way analysis of variance followed by Tukey's multiple comparison test, and resistance factors. Taking into account the 13 independent tests of the different drugs, the Bonferroni-corrected threshold for significance is $p<0.00385$

and melarsoprol itself, though not all the differences were statistically significant $(p<0.004$ in Anova one-way analysis of variance followed by Tukey's multiple comparison test; Table 1). On the other hand, STIB900-P was cross-resistant to the adenine analog aminopurinol (not a AT1 substrate [44]) whereas STIB900-M was not (Table 1). Neither line was resistant ( $p<0.004$ in Anova plus Tukey's test) to suramin, nifurtimox, fexinidazole, or phenylarsine oxide, a hydrophobic arsenical that diffuses across the plasma membrane [13]. Surprisingly, both STIB900-M and STIB900-P were significantly hypersensitive to eflornithine (difluoromethylornithine, DFMO). This unexpected result is consistent with the observation that eflornithine resistance caused by loss of the amino acid transporter TbAAT6 was accompanied by hypersensitivity to pentamidine [45]. TbAAT6 was not overexpressed in the resistant lines (see below). The in vitro population doubling times without drug were $9.4 \pm 0.3 \mathrm{~h}$ and did not significantly differ between the tree lines $(p>0.05$ in Anova one-way analysis of variance followed by Tukey's multiple comparison test).

\section{Transcriptomic profiling indicates loss of expression of transporter genes}

Quantitative transcriptomics served as a first tool to investigate the strong MPXR phenotype of $T$. $b$. rhodesiense STIB900-P and STIB900-M at the molecular level. For this purpose we used the 'spliced leader trapping' (SLT) adaptation of Illumina RNA-Seq [29], exploiting the fact that all trypanosomal mature mRNAs carry the same $39 \mathrm{nt}$ leader sequence spliced in-trans to their $5^{\prime}$ end, a peculiarity of trypanosomatids [46]. SLT is optimally suited to quantify steady-state mRNA levels by counting the number of reads per transcript because all the reads stem from the transcription start sites. Figure 1 shows one of two independent experiments; all data are included in the supplementary Excel file Graf_S1.xlsx. Overall, there was very little variance between the drug-resistant lines and their sensitive parent (Fig. 1a). Four genes were overexpressed in STIB900-P compared to STIB900 and STIB900$\mathrm{M}$, all of which are neighboring genes on chromosome 6 encoding for VSGs. This confirms that antigenic variation also takes place in vitro, but new variants rarely become fixed in the population because of continuous dilution of the growing cultures. Apart from these VSG, no genes were significantly overexpressed in the resistant $T$. $b$. rhodesiense lines-including TbMRPA, an export pump that confers resistance to melarsoprol when overexpressed ectopically [47, 48]. The adenosine transporter $A T 1$ and six adjacent genes on the telomere of chromosome 5 [49] were not expressed above detection limit in STIB900-M (Fig. 1b). This is in agreement with the reported absence of $A T 1$ in STIB900-M [24] and is indicative of a larger deletion at the $A T 1$ locus. The aquaglyceroporin $A Q P 2$ appeared not to be expressed above detection limit in either resistant line (Fig. 1c). However, the automated mapping of the reads [29] was ambiguous because of the high degree of similarity between $A Q P 2$ and $A Q P 3$ at their $5^{\prime}$ ends 

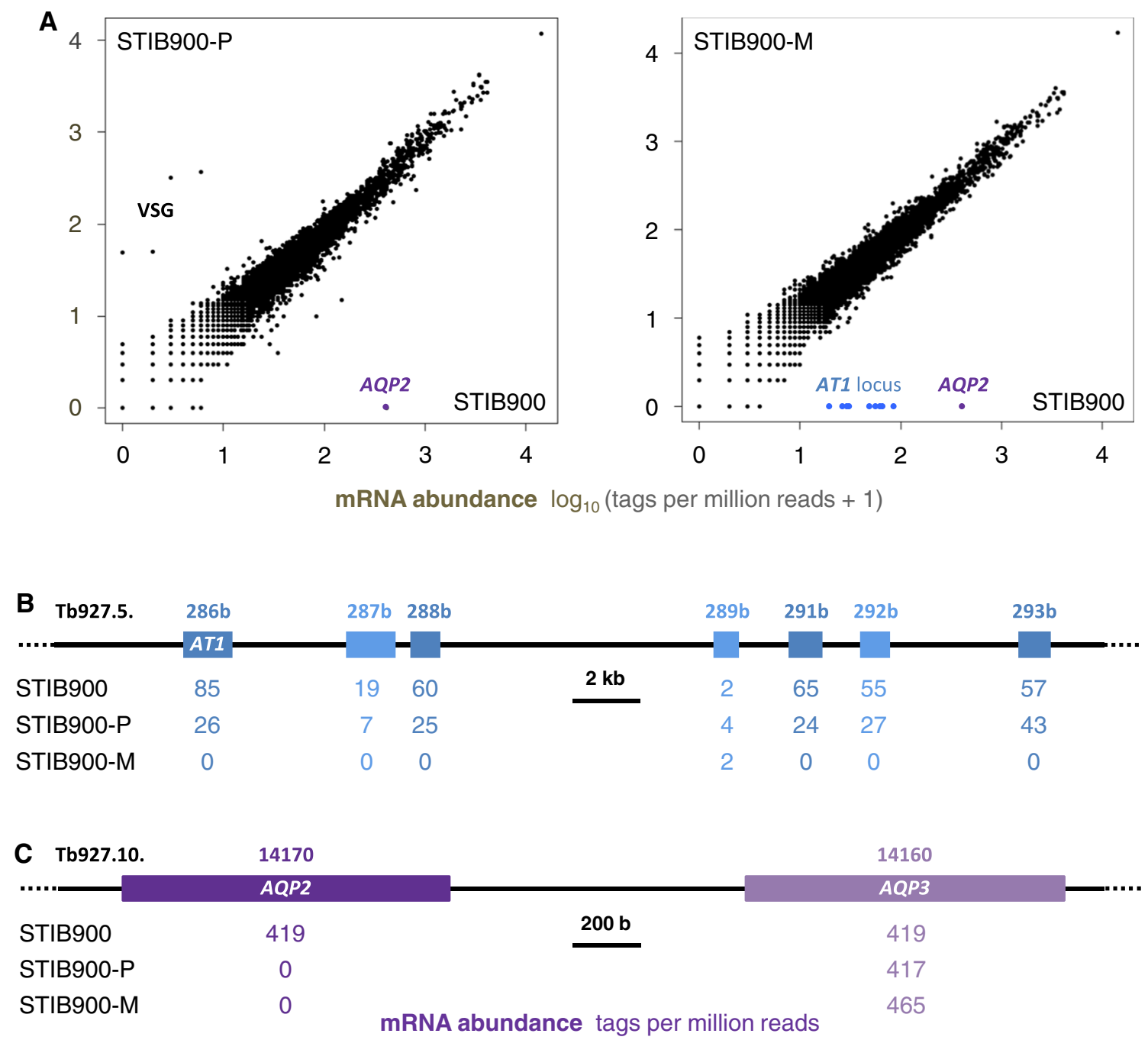

Fig. 1 Comparative transcriptomics. a Scatter plots of normalized read counts from RNA sequencing data using the spliced leader trapping protocol. The increment of one allowed logarithmic representation also for genes that had zero sequence tags. Genes that are

(Fig. 2). The short Illumina reads were manually mapped to $A Q P 3$ since the deletion of $A Q P 2$ was confirmed by the longer 454 reads (Fig. 2). Apart from AT1 plus adjacent genes and $A Q P 2$, no genes were significantly under expressed in the resistant lines.

\section{A reference genome sequence of the $T$. $b$. rhodesiense drug-sensitive parent}

Before exploring the mutations underlying the strong MPXR phenotype of STIB900-P and STIB900-M by comparative genomics, we had to generate a good-quality draft genome of the susceptible parent $T$. $b$. rhodesiense STIB900. Genomic DNA was isolated from bloodstreamform trypanosomes grown in mice. The obtained gDNA was verified to be free of mouse DNA by PCR with primers not expressed in the resistant lines are indicated. Note the VSG switch in STIB900-P. b View of the AT1 locus with read counts per gene. c View of the $A Q P 2 / A Q P 3$ tandem locus with read counts per gene

for mouse GAPDH. Paired-end Illumina reads generated on the HiSeq platform were mapped to the core chromosomes of $T$. b. brucei TREU927 with an average coverage of 53 fold. The vast majority of gene models (9692 of 9722) were transferred from T. b. brucei TREU927 to the assembled T. $b$. rhodesiense STIB900 genome, identifying a total of 112,565 high-quality single-nucleotide polymorphisms (SNP) between these two genomes. In proteincoding regions there were 46,453 SNPs, of which 19,575 non-synonymous. As expected, the assembled $T . b$. rhodesiense STIB900 genome contained the SRA gene (serum resistance-associated; Tb927.9.17380), whose product neutralizes ApoL1, the trypanolytic factor of human serum that protects humans from infection by T. b. brucei. The genome reference strain $T$. b. brucei TREU927 contains a dysfunctional SRA ortholog $[50,51]$. 
A

STIB900-P
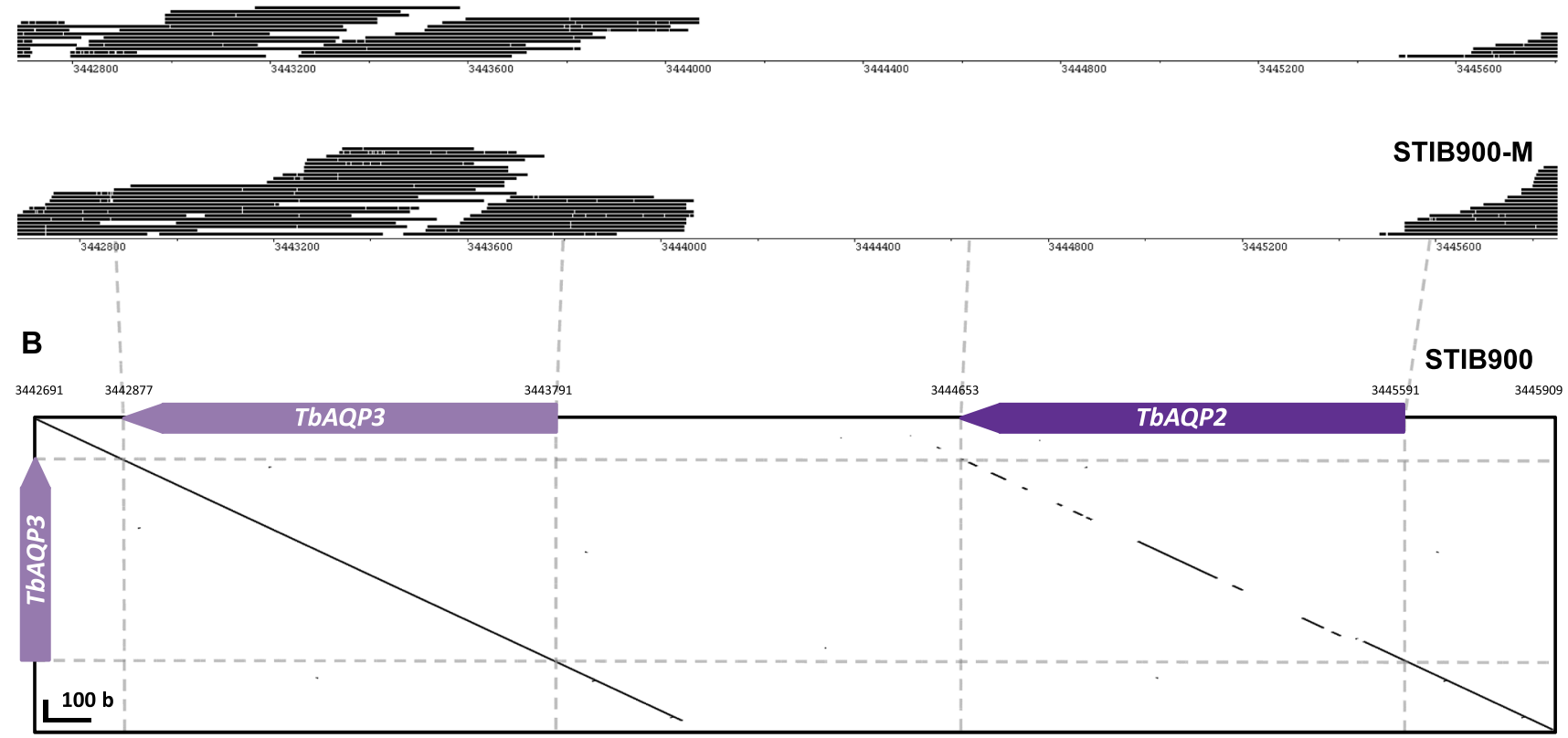

Fig. 2 Loss of $A Q P 2$ from the resistant lines. a Roche-454 sequencing reads generated from genomic DNA of STIB900-P and STIB900$M$ mapped to the reference sequence of STIB900 as visualized with BamView [59]. The gene $A Q P 2$ is missing in both STIB900-P and STIB900-M. b The tandem nature of the AQP2/AQP3 locus on

\section{Comparative genomics confirms the loss of transporter genes}

The assembled genome sequence of $T$. $b$. rhodesiense STIB900 was used as a reference to identify mutations in the resistant derivatives STIB900-P and STIB900-M. Genomic DNA was isolated from bloodstream-form trypanosomes harvested from infected mice. The known absence of ATl from STIB900-M was used for diagnostic PCR of the purified gDNA to verify that there had been no contaminations. Roche-454 sequencing libraries were generated for all three lines, obtaining $\sim 1.5$ million highquality reads for each genome, corresponding to a 20 -fold coverage (Table S1). The Roche/454 reads from STIB900$\mathrm{M}$ and STIB900-P were mapped to the assembled STIB900 genome with an overall coverage of $83 \%$ (DP4 $\geq 5$ ). SNPs and indels were identified with SAMtools and with selfdeveloped Perl scripts. All identified SNPs, indels, and gene deletions were inspected manually using Artemis [37]. Overall, there were remarkably few mutations in STIB900-M and STIB900-P relative to their parent STIB900 (Table 2). Only one coding point mutation was found in both resistant lines (in the gene $U B P 1$, see below). Both lines carried a deletion of about $1.8 \mathrm{~kb}$ at the $A Q P 2 /$ $A Q P 3$ locus (Fig. 2a), causing loss of the aquaglyceroporin $A Q P 2$ but not of $A Q P 3$. The deletion was confirmed by PCR on genomic DNA followed by sequencing of the chromosome 10 as illustrated by a dot plot of the STIB900 reference sequence from (a) on the $x$ axis vs. the sequence of $A Q P 3$ on the $y$ axis (made with GEPARD [60] and a word length of 10). The genes $A Q P 2$ and $A Q P 3$ are highly similar (82\% global identity) as depicted by the diagonal on the right

products (not shown). T. b. rhodesiense STIB900-M had not only lost the gene ATl (Tb927.5.286b), as previously published [24], but a whole region of over $25 \mathrm{~kb}$ encompassing ATI and the adjacent genes Tb927.5.288b (ribulose-phosphate 3-epimerase, putative), Tb927.5.289b (hypothetical protein), Tb927.5.291b (variant surface glycoprotein-related), and Tb927.5.292b (hypothetical protein).

\section{A point mutation that renders TbAT1 non- functional is heterozygous in STIB900-P}

Trypanosoma brucei rhodesiense STIB900-P still possessed $A T 1$ but the gene contained a non-synonymous substitution, G1288C, resulting in the mutation of glycine $^{430}$ to arginine. The identified point mutation was confirmed with Sanger sequencing and by restriction digest of the ATl PCR product, since the mutation generated an endonuclease NruI site (tcgcga; Fig. 3). The facts that the AT1 PCR products from genomic DNA of STIB900-P (1) were not digested completely (Fig. 3), and (2) after cloning and sequencing did not all contain the $\mathrm{C}$ at position 1288 (not shown), indicate that STIB900-P is heterozygous for the mutation G1288C. The AT1-G430R mutant was functionally characterized in $T$. b. brucei $\mathrm{B} 48$, a mutant which lacks high-affinity transport of melarsoprol and pentamidine [16, 52]. B48 bloodstream-form cells were 
Table 2 SNP statistics

\begin{tabular}{|c|c|c|c|c|c|c|}
\hline & \multicolumn{3}{|c|}{ STIB900-M vs. STIB900 } & \multicolumn{3}{|c|}{ STIB900-P vs. STIB900 } \\
\hline & Overall & CDS & NS & Overall & CDS & NS \\
\hline Chr1 & 0 & 0 & 0 & 2 & 0 & 0 \\
\hline Chr2 & 0 & 0 & 0 & 0 & 0 & 0 \\
\hline Chr3 & 0 & 0 & 0 & 1 & 0 & 0 \\
\hline Chr4 & 0 & 0 & 0 & 0 & 0 & 0 \\
\hline Chr5 & 2 & 0 & 0 & 1 & Tb927.5.286b & G430R \\
\hline Chr6 & 1 & 0 & 0 & 0 & 0 & 0 \\
\hline Chr7 & 0 & 0 & 0 & 1 & 0 & 0 \\
\hline Chr8 & 1 & 0 & 0 & 0 & 0 & 0 \\
\hline Chr9 & 6 & 0 & 0 & 5 & Tb927.9.8310* & D31G \\
\hline Chr10 & 0 & 0 & 0 & 0 & 0 & 0 \\
\hline Chr11 & 2 & Tb927.11.500 & $\mathrm{R} 131 \mathrm{~L}$ & 4 & Tb927.11.500 & $\mathrm{R} 131 \mathrm{~L}$ \\
\hline Total & 12 & 1 & 1 & 14 & 3 & 3 \\
\hline
\end{tabular}

Single-nucleotide polymorphisms between the drug-resistant $T$. b. rhodesiense lines and their parent STIB900 (CDS coding sequence, NS nonsynonymous)

* This gene is no longer part of TriTrypDB

transfected with wildtype $A T 1, \mathrm{G} 430 \mathrm{R}$ mutant $A T 1$, and empty vector as a control. Expression of wildtype AT1 strongly sensitized the B48 transfectants to melarsoprol, pentamidine, and diminazene aceturate $\left(\right.$ Berenil $\left.^{\circledR}\right)$, whereas G430R mutant AT1 did not (Fig. 4). This demonstrates that the point mutation renders AT1 non-functional with respect to drug transport.

\section{Concomitant deletion of AQP2 and AT1 does not phenocopy the high-level MPXR}

The contributions to MPXR of either $A Q P 2$ or $A T 1$ alone have been extensively studied $[11,53]$. To try and phenocopy T. b. rhodesiense STIB900-P and STIB900-M, respectively, we have generated heterozygous and homozygous $T$. b. brucei at 1 null lines (Supplementary Figure S1) in a aqp2/aqp3 null background [17]. The four lines 2T1 (parental), 2T1-aqp2/aqp3 ${ }^{-/-}$, 2T1-aqp2/ $a q p 3^{-/-}-a t 1^{+/-}$, and 2T1-aqp2/aqp3 $3^{-/-}-a t 1^{-/-}$were characterized regarding their growth and drug sensitivities. The four lines grew equally well in vitro with population doubling times of around $7.0 \pm 0.5 \mathrm{~h}(p>0.05$ in Anova one-way analysis of variance followed by Tukey's multiple comparison test), indicating that simultaneous loss of $A T 1$, $A Q P 2$, and $A Q P 3$ does not bear a fitness cost in vitro. In agreement with previous findings [17], the $\mathrm{IC}_{50}$ of pentamidine increased by about 15 fold in the absence of $A Q P 2$. Heterozygous deletion of $A T 1$ further increased the resistance factor to 20, homozygous deletion to 35 (Fig. 5). The susceptibilities of the four strains to melarsoprol did not follow such a pronounced pattern, with moderated resistance factors of 2-2.5 (Fig. 5). Clearly, the MPXR resistance factors obtained through simultaneous knock-out of $A T 1$ and $A Q P 2$ were below those of $T$. $b$. rhodesiense STIB900-M and STIB900-P (Table 1), indicating that further mutations must contribute to the high-level MPXR phenotype observed in those lines.

\section{Testing of the RNA-binding protein UBP1 as a new candidate resistance gene}

In view of the very small number of non-synonymous SNPs (Table 2), the finding that the two independently selected lines, STIB900-P and STIB900-M, were homozygous for the same point mutation, $\operatorname{Arg}^{131}$ to Leu, in the uridine-rich-binding protein 1 (UBP1, Tb927.11.500; Table 2) was of particular interest. UBP1 is an RNAbinding protein with a single, conserved RNA recognition motif (RRM, Fig. 6a), whose structure has been determined for its ortholog in Trypanosoma cruzi [54]. Arg $^{131}$ of TbUBP1 corresponds to Arg ${ }^{113}$ of TcUBP1 in T. cruzi and lies within the B4-B5 hairpin of the RRM (Fig. 6a) that is involved in RNA binding [54]. To test the impact of the mutation R131L on TbUBP1 function, we first generated two stable $T$. $b$. brucei $2 \mathrm{~T} 1$ cell lines overexpressing either GFP-UBP1-wt or GFP-UBP1-R131L at a single specific locus within the rRNA spacer in a tetracycline (Tet)-inducible manner [42]. Both lines still possessed the endogenous UBP1 alleles. Overexpression of GFP-UBP1wt for longer than $24 \mathrm{~h}$ caused a strong growth defect 

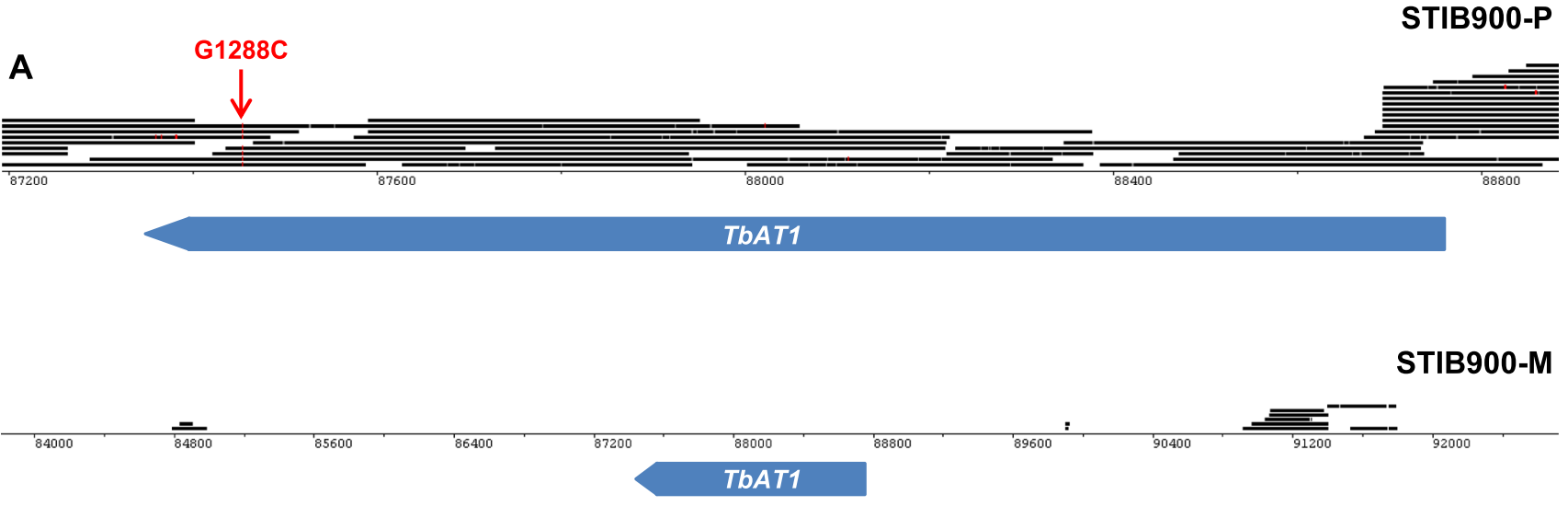

B

\section{T. b. rhodesiense STIB900}

T. b. rhodesiense STIB900-P
ACAGCTGGCGAGC ACAGCTCGCGAGC

Nrul
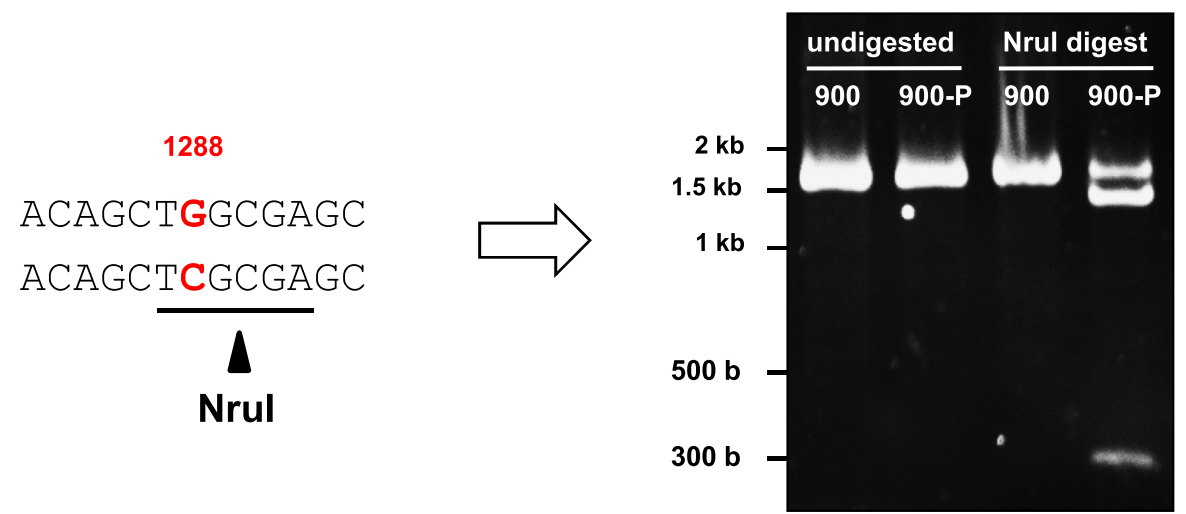

Fig. 3 Loss or mutation of $A T 1$ in the resistant lines. a Genomic 454 reads of STIB900-P and STIB900-M mapped to STIB900 visualized for the AT1 locus on chromosome 5 using BamView [59] (smaller scale for STIB900-M). There is a deletion of AT1 in STIB900-M and a coding point mutation in STIB900-P (red). b AT1 PCR products

(Supplementary Figure S2A), which is in agreement with previous findings [55]. Interestingly, overexpression of GFP-UBP1-R131L did not cause a growth phenotype (Figure S2A). However, the GFP-UBP1-R131L overexpressing trypanosomes were slightly but significantly hypersensitive to pentamidine as compared to non-induced cells ( $\mathrm{IC}_{50}$ of $1.2 \mathrm{nM}$ vs. $2.7 \mathrm{nM}$; Figure $\left.\mathrm{S} 2 \mathrm{~B}\right)$. We do not have an explanation for this counter-intuitive resultwhich has to be interpreted with caution due to the lack of a control since overexpression of wildtype UBP1 was toxic to the trypanosomes. Attempting to test the role of the identified mutation in a more physiological context, we introduced the mutant gene UBPI-R131L in T. b. brucei $2 \mathrm{~T} 1$ cells in situ. A blasticidin resistance gene $(B S D)$ was added to the $5^{\prime}$ UTR of the UBPI gene together with the mutation encoding for Leu ${ }^{131}$ (Supplementary Figure S3A). Antibiotic pressure allowed selection of two homozygous clones (designated $\mathrm{H}$ and $\mathrm{L}$ ) without the need for a second round of transfection (Fig. 6b, Supplementary Figure S3B). However, neither of the two clones exhibited
(1636 bp) were amplified from genomic DNA of STIB900 and STIB900-P, and digested with the endonuclease NruI. G1288C mutant alleles are cut to fragments of 1339 and $257 \mathrm{bp}$. STIB900-P appears to be heterozygous for the mutation

a significantly reduced sensitivity to melarsoprol or pentamidine as compared to parental 2T1 cells (Fig. 6c).

\section{Discussion}

Cross-resistance of African trypanosomes to melarsoprol and pentamidine (MPXR) is a well-known phenomenon [11]. Here we perform in-depth phenotypic and genotypic profiling of two lab-derived T. $b$. rhodesiense mutants with most pronounced MPXR phenotypes: STIB900-P, selected with pentamidine, and STIB900-M, selected with melarsoprol. The two independently selected lines exhibited similar-but not identical (Table 1)—resistance profiles, extending MPXR to other diamidines and adenosine analogs, but not to suramin or nifurtimox.

Very few mutations had become fixed in the drug-selected lines in the 2 years' course of selection (Table 2), and very few genes were differentially expressed in the resistant lines compared to the sensitive parent (Fig. 1). 


\section{Pentamidine}
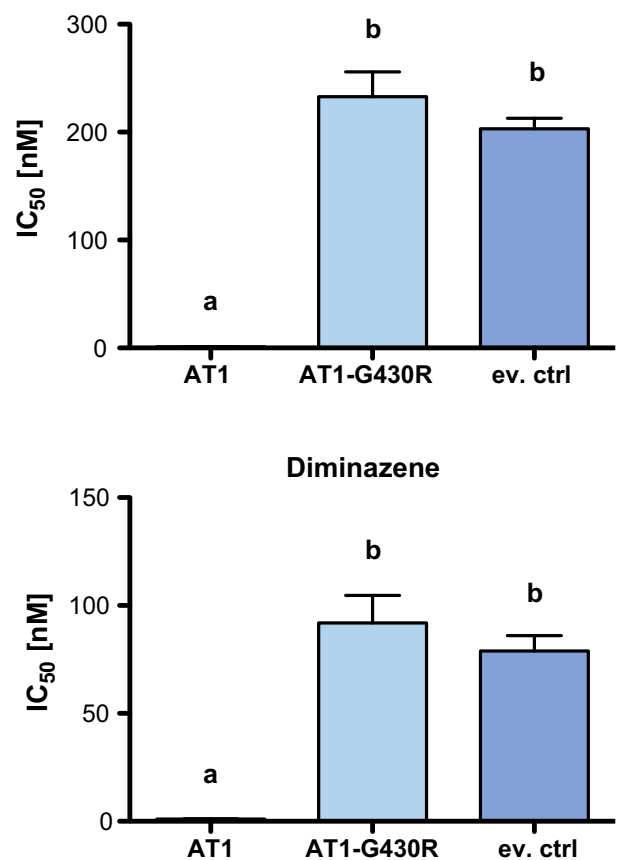

Fig. 4 Functional characterization of G430R mutant $A T 1$. The genes $A T 1, A T 1-G 430 R$, and an empty vector control (ev. ctrl) were expressed in the T. b. brucei B48 mutant [16]. Transfectant clones were tested in vitro for their sensitivity to the known AT1 substrates pentamidine, cymelarsan, and diminazene. Phenylarsine oxide (PAO)

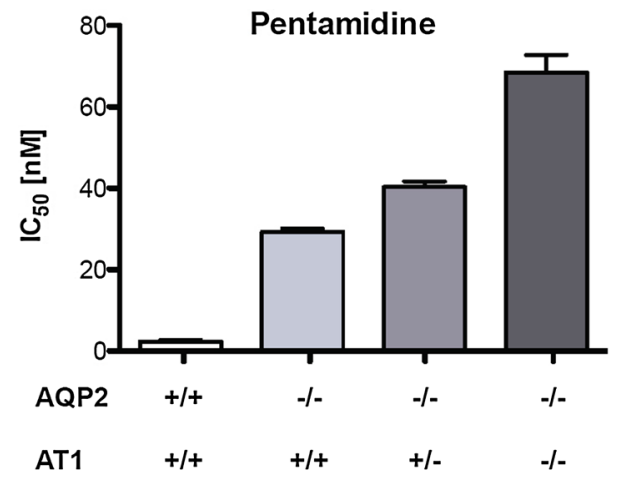

Fig. $5 A T 1 / A Q P 2$ double knock-out in $T$. brucei. In vitro drug sensitivities were determined for pentamidine and melarsoprol on parental $2 \mathrm{~T} 1$ cells, 2T1-aqp2 $2^{-/-}, 2 \mathrm{~T} 1-a q p 2^{-/-}-A T 1^{+/-}$, and 2T1-

The only striking difference was the complete absence of RNA-Seq reads for AQP2 in STIB900-M and STIB900-P, and for $A T 1$ and neighboring genes in STIB900-M. Both STIB900-M and STIB900-P carried a deletion of the $A Q P 2$ locus (Fig. 2). This 'natural knock-out' of $A Q P 2$ was probably due to homologous recombination with the neighboring, highly similar gene $A Q P 3$ (Fig. 2), accompanied by loss of genetic material. Cases of truncation or chimerization of $A Q P$ genes in drug-resistant $T$. brucei had

\section{Cymelarsan}

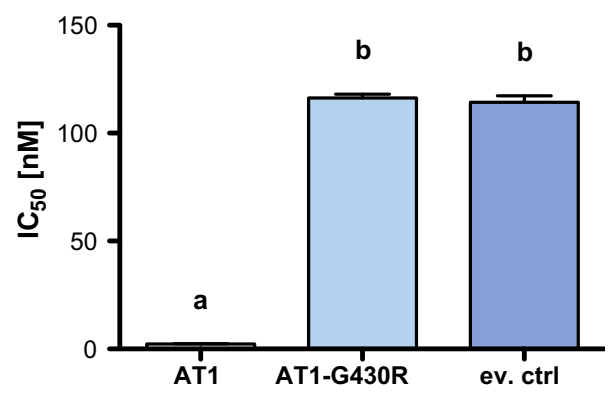

PAO

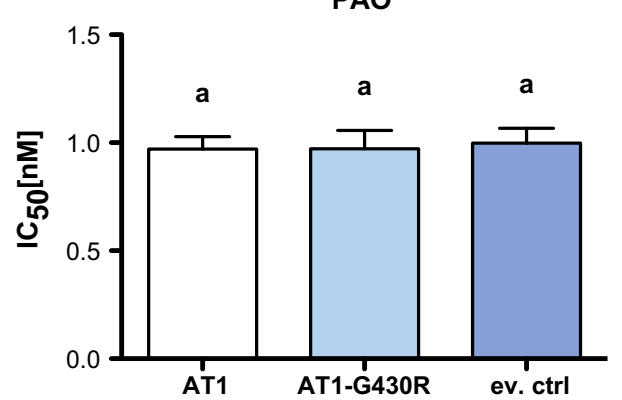

was included as a negative control that is not a AT1 substrate. The $\mathrm{IC}_{50}$ values are from 5 to 8 independent experiments; error bars are standard errors of the mean. The small letters indicate significance groups $(p<0.05)$ as determined by Anova one-way analysis of variance followed by Tukey's multiple comparison test

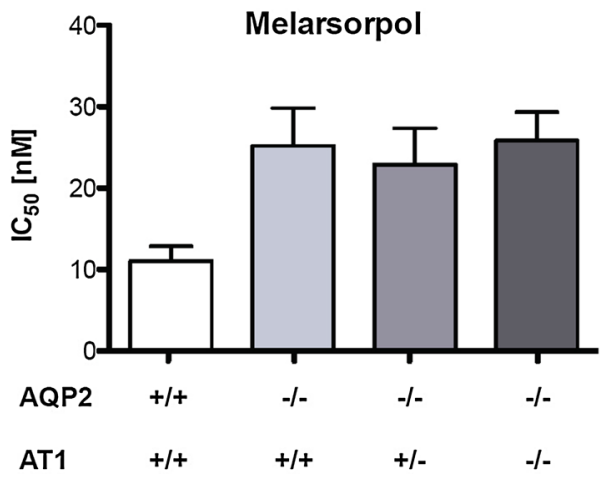

$a q p 2^{-1-}-a t 1^{-1-}$. Bars are the average $\mathrm{IC}_{50}$ of at least four independent experiments, each performed in triplicate. Error bars indicate the standard error of the mean

been reported previously $[17,20,21,56] . T$. $b$. rhodesiense STIB900-M also carried a large deletion on chromosome five encompassing $A T 1$ and six adjacent genes (Fig. 3), a gene loss possibly facilitated by their telomeric location. STIB900-P had a non-synonymous point mutation in ATl changing $\mathrm{Gly}^{430}$ to $\mathrm{Arg}$ (Fig. 3). Expression of wildtype and G430R-mutant $A T 1$ in a $T$. b. brucei loss of transport mutant demonstrated that the AT1-G430R did not transport melarsoprol or diamidines (Fig. 4). The finding that 
A

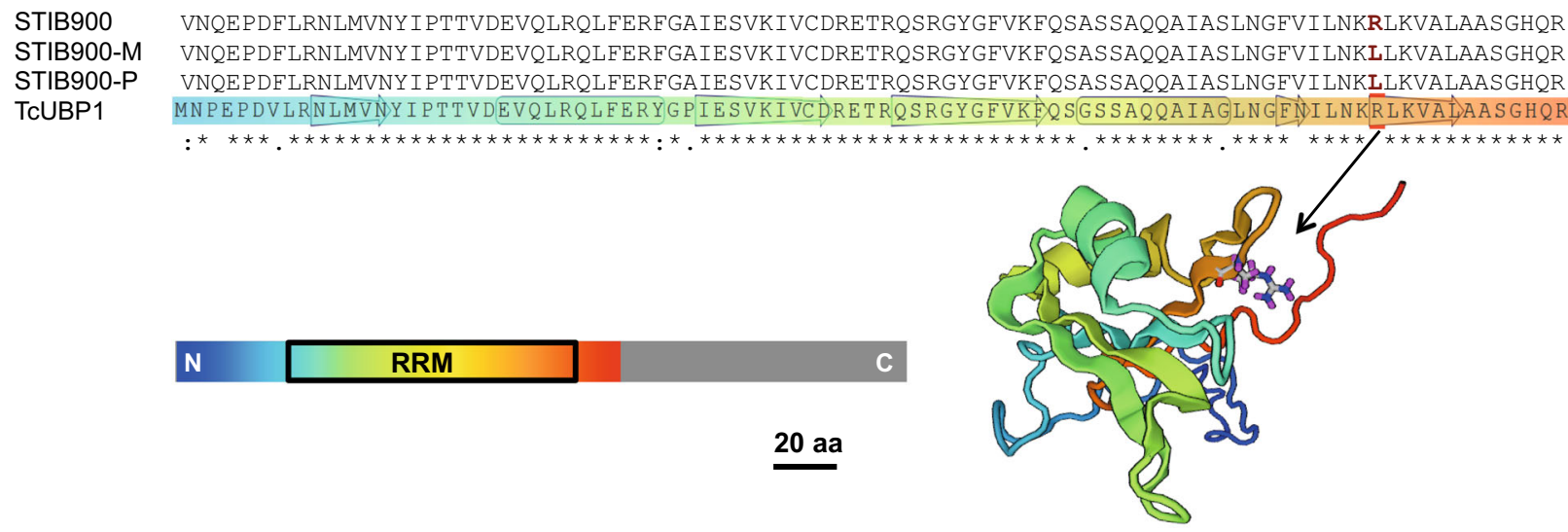

B
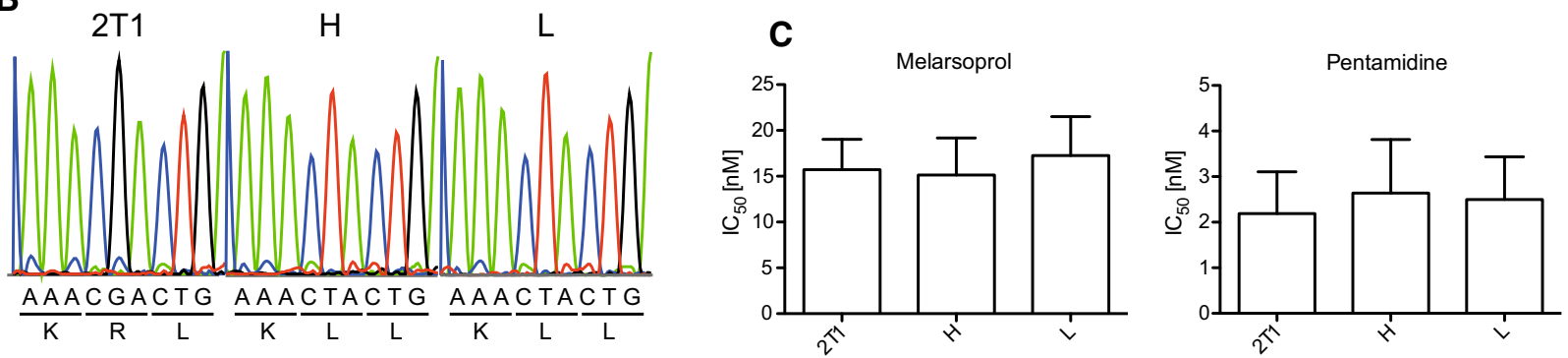

Fig. 6 In situ mutation of $U B P 1$ in $T$. b. brucei. a Alignment of the predicted RNA recognition motif (RRM) of UBP1 from STIB900, STIB900-M, and STIB900-P alongside Trypanosoma cruzi UBP1. The mutation of the conserved Arg ${ }^{131}$ to Leu is highlighted in the alignment and $\operatorname{Arg}^{131}$ is indicated with an arrow in the published structure of TcUBP1. The full length TcUBP1 is 224 aa but a truncated version (1-139) including the RRM (35-126) was used to determine the structure [54]. Swiss model [61] was used to visualize the structure. b Sanger sequencing electropherograms of $U B P 1$

amplified from genomic DNA of parental $2 \mathrm{~T} 1$ cells and two transgenic clones $\mathrm{H}$ and $\mathrm{L}$, showing the nucleotides that encode amino acids 130-132. c In vitro drug sensitivities $\left(\mathrm{IC}_{50} \pm\right.$ standard deviation; $n=12$ ) for melarsoprol and pentamidine determined with the Alamar blue assay for parental 2T1 cells and the two transgenic clones $\mathrm{H}$ and $\mathrm{L}$. There was no significant difference in drug sensitivity between the three lines (Anova one-way analysis of variance followed by Tukey's multiple comparison test)

STIB900-P is heterozygous for the mutation might explain its milder MPXR phenotype than STIB900-M (Table 1). These results are the best evidence to date that the MPXR models developed in the non-human infective trypanosome $T$. $b$. brucei hold true in T. $b$. rhodesiense, the causative agent of sleeping sickness in East Africa, and that selection for high-level resistance to melarsoprol and pentamidine can lead to loss of both known drug transporters.

To investigate the combined contribution of $A Q P 2$ and ATl to drug sensitivity, we constructed $T$. $b$. brucei heteroand homozygous at 1 null mutants in a aqp2/aqp3 null background. The effects of the mutations were additive regarding pentamidine sensitivity, with a maximal resistance factor of 35 for the double null mutant of at 1 and aqp2 (Fig. 5). The phenotypes with respect to melarsoprol were less pronounced, as the loss of $A T 1$ did not further increase the melarsoprol resistance of the aqp 2 null mutant (Fig. 5). The conclusion from these experiments is that

concomitant deletion of $A Q P 2$ and AT1 does not completely phenocopy the strong MPXR phenotypes of $T$. $b$. rhodesiense STIB900-M and STIB900-P, demonstrating that further genes must be involved. Only one additional gene was affected by a mutation in both resistant lines: the RNA-binding protein $U B P 1$.

Trypanosoma brucei rhodesiense STIB900-M and STIB900-P both carried the mutation R131L in UBP1, which was absent in the parent and must have been acquired by the resistant lines independently. The precise function of UBP1 is unknown, but it has been implicated in regulation of mRNA levels [55]. Based on the alignment to TcUBP1 from T. cruzi, $\operatorname{Arg}^{131}$ of TbUBP1 is predicted to be critical for RNA binding (Fig. 6a), suggesting that $\mathrm{Leu}^{131}$ may impair TbUBP1 function. Complete loss of UBP1 function may be lethal, as indicated by RNAi-mediated knock-down of $U B P 1$ and $U B P 2$ [55]. TcUBP1 is a cytoplasmic protein in epimastigotes of $T$. cruzi [57]. 
Intriguingly, TcUBP1 was shown to accumulate in the nucleus when the trypanosomes were under arsenite stress, and mutations affecting RNA binding prevented nuclear accumulation of TcUBP1 [57]. TbUBP2, which shares $73 \%$ global identity with $T b U B P 1$, had appeared as a secondary hit in a genome-wide RNAi screen for pentamidine resistance in $T$. brucei [58]. While such previous findings may support a possible role of UBP1 in drug resistance, our reverse genetic approaches did not. Nonphysiological overexpression of UBP-Leu ${ }^{131}$-GFP in $T$. $b$. brucei bloodstream forms even caused a slight hypersensitivity to pentamidine (Figure S2B). However, this needs to be interpreted cautiously since, in agreement with a previous report [55], overexpression of the 'wildtype' UBP1-GFP fusion protein was lethal (Figure S2A). More physiological in situ expression of the mutant $U B P 1$ in $T$. b. brucei did not affect the sensitivity to melarsoprol or pentamidine (Fig. 6c). In summary, if the mutation $\mathrm{Arg}^{131}$ to Leu in UBP1 contributes to drug resistance at all, then it does so only in the context of the described loss of AQP2 and/or AT1.

Acknowledgments We would like to thank Hansjörg Keller for mouse cDNA and André Schneider for the BiP antibody. We are very grateful to Roche for a $10 \mathrm{~GB}$ Sequencing Award that has granted us free access to the Roche-454 platform and to the Emilia GuggenheimSchnurr Foundation for financial support with spliced leader trapping. This research was supported by the Swiss National Science Foundation, by the MRC and Department for International Development, UK under the MRC/DFID Concordat agreement (grant MR/K000500/1 to DH), and by the Wellcome Trust (Grant 100320/Z/12/Z; Investigator Award to DH). FEG and PL received additional support by the Mathieu Stiftung Basel and the Freiwillige Akademische Gesellschaft Basel. PL was additionally supported by the Emilia GuggenheimSchnurr Foundation. Support for the STIB900 Illumina data was provided to the NIH R21 Grant AI094615-01 awarded to AC.

Open Access This article is distributed under the terms of the Creative Commons Attribution 4.0 International License (http:// creativecommons.org/licenses/by/4.0/), which permits unrestricted use, distribution, and reproduction in any medium, provided you give appropriate credit to the original author(s) and the source, provide a link to the Creative Commons license, and indicate if changes were made.

\section{References}

1. El-Sayed NM, Hegde P, Quackenbush J, Melville SE, Donelson JE (2000) The African trypanosome genome. Int J Parasitol 30(4):329-345

2. Berriman M, Ghedin E, Hertz-Fowler C, Blandin G, Renauld H, Bartholomeu DC, Lennard NJ, Caler E, Hamlin NE, Haas B, Bohme U, Hannick L, Aslett MA, Shallom J, Marcello L, Hou L, Wickstead B, Alsmark UC, Arrowsmith C, Atkin RJ, Barron AJ, Bringaud F, Brooks K, Carrington M, Cherevach I, Chillingworth TJ, Churcher C, Clark LN, Corton CH, Cronin A, Davies RM,
Doggett J, Djikeng A, Feldblyum T, Field MC, Fraser A, Goodhead I, Hance Z, Harper D, Harris BR, Hauser H, Hostetler J, Ivens A, Jagels K, Johnson D, Johnson J, Jones K, Kerhornou AX, Koo H, Larke N, Landfear S, Larkin C, Leech V, Line A, Lord A, Macleod A, Mooney PJ, Moule S, Martin DM, Morgan GW, Mungall K, Norbertczak H, Ormond D, Pai G, Peacock CS, Peterson J, Quail MA, Rabbinowitsch E, Rajandream MA, Reitter C, Salzberg SL, Sanders M, Schobel S, Sharp S, Simmonds M, Simpson AJ, Tallon L, Turner CM, Tait A, Tivey AR, Van Aken S, Walker D, Wanless D, Wang S, White B, White O, Whitehead S, Woodward J, Wortman J, Adams MD, Embley TM, Gull K, Ullu E, Barry JD, Fairlamb AH, Opperdoes F, Barrell BG, Donelson JE, Hall N, Fraser CM, Melville SE, El-Sayed NM (2005) The genome of the African trypanosome Trypanosoma brucei. Science 309(5733):416-422

3. Brun R, Blum J, Chappuis F, Burri C (2010) Human African trypanosomiasis. Lancet 375:148-159

4. Kennedy PG (2008) The continuing problem of human African trypanosomiasis (sleeping sickness). Ann Neurol 64(2):116-126. doi:10.1002/ana.21429

5. Kibona SN, Matemba L, Kaboya JS, Lubega GW (2006) Drugresistance of Trypanosoma $b$. rhodesiense isolates from Tanzania. Trop Med Int Health 11(2):144-155. doi:10.1111/j.1365-3156. 2005.01545.x

6. Matovu E, Enyaru JC, Legros D, Schmid C, Seebeck T, Kaminsky $\mathrm{R}$ (2001) Melarsoprol refractory T. b. gambiense from Omugo, north-western Uganda. Trop Med Int Health 6(5):407-411

7. Stewart ML, Krishna S, Burchmore RJ, Brun R, de Koning HP, Boykin DW, Tidwell RR, Hall JE, Barrett MP (2005) Detection of arsenical drug resistance in Trypanosoma brucei with a simple fluorescence test. Lancet 366(9484):486-487. doi:10.1016/ S0140-6736(05)66793-1

8. Robays J, Nyamowala G, Sese C, Mesu Betu Ku, Kande V, Lutumba P, Van der Veken W, Boelaert M (2008) High failure rates of melarsoprol for sleeping sickness, Democratic Republic of Congo. Emerg Infect Dis 14(6):966-967. doi:10.3201/eid1406. 071266

9. Mäser P, Wittlin S, Rottmann M, Wenzler T, Kaiser M, Brun R (2012) Antiparasitic agents: new drugs on the horizon. Curr Opin Pharmacol 12(5):562-566. doi:10.1016/j.coph.2012.05.001

10. Rollo IM, Williamson J (1951) Acquired resistance to 'melarsen', tryparsamide and amidines in pathogenic trypanosomes after treatment with 'melarsen' alone. Nature 167(4239):147-148

11. Baker N, de Koning HP, Mäser P, Horn D (2013) Drug resistance in African trypanosomiasis: the melarsoprol and pentamidine story. Trends Parasitol 29(3):110-118. doi:10.1016/j.pt.2012.12.005

12. de Koning HP, Jarvis SM (1999) Adenosine transporters in bloodstream forms of Trypanosoma brucei brucei: substrate recognition motifs and affinity for trypanocidal drugs. Mol Pharmacol 56(6): 1162-1170

13. Carter NS, Fairlamb AH (1993) Arsenical-resistant trypanosomes lack an unusual adenosine transporter. Nature 361:173-175

14. Mäser P, Sütterlin C, Kralli A, Kaminsky R (1999) A nucleoside transporter from Trypanosoma brucei involved in drug resistance. Science 285(5425):242-244

15. de Koning H (2001) Uptake of pentamidine in Trypanosoma brucei is mediated by three distinct transporters: implications for cross-resistance with arsenicals. Mol Pharmacol 59(3):586-592

16. Bridges DJ, Gould MK, Nerima B, Mäser P, Burchmore RJ, de Koning HP (2007) Loss of the high-affinity pentamidine transporter is responsible for high levels of cross-resistance between arsenical and diamidine drugs in African trypanosomes. Mol Pharmacol 71(4):1098-1108. doi:10.1124/mol.106.031351

17. Baker N, Glover L, Munday JC, Aguinaga Andres D, Barrett MP, de Koning HP, Horn D (2012) Aquaglyceroporin 2 controls susceptibility to melarsoprol and pentamidine in African 
trypanosomes. Proc Natl Acad Sci USA 109(27):10996-11001. doi:10.1073/pnas.1202885109

18. Munday JC, Eze AA, Baker N, Glover L, Clucas C, Aguinaga Andres D, Natto MJ, Teka IA, McDonald J, Lee RS, Graf FE, Ludin P, Burchmore RJ, Turner CM, Tait A, MacLeod A, Maser P, Barrett MP, Horn D, De Koning HP (2014) Trypanosoma brucei aquaglyceroporin 2 is a high-affinity transporter for pentamidine and melaminophenyl arsenic drugs and the main genetic determinant of resistance to these drugs. J Antimicrob Chemother 69(3):651-663. doi:10.1093/jac/dkt442

19. Matovu E, Geiser F, Schneider V, Mäser P, Enyaru JCK, Kaminsky R, Gallati S, Seebeck T (2001) Genetic variants of the TbAT1 adenosine transporter from African trypanosomes in relapse infections following melarsoprol therapy. Mol Biochem Parasitol 117(1):73-81

20. Graf FE, Ludin P, Wenzler T, Kaiser M, Brun R, Pyana PP, Büscher P, de Koning HP, Horn D, Mäser P (2013) Aquaporin 2 mutations in Trypanosoma brucei gambiense field isolates correlate with decreased susceptibility to pentamidine and melarsoprol. PLoS Negl Trop Dis 7(10):e2475. doi:10.1371/ journal.pntd.0002475PNTD-D-13-00953

21. Graf FE, Baker N, Munday JC, de Koning HP, Horn D, Mäser $P$ (2015) Chimerization at the AQP2-AQP3 locus is the genetic basis of melarsoprol-pentamidine cross-resistance in clinical Trypanosoma brucei gambiense isolates. Int J Parasitol Drugs Drug Resist 5(2):65-68. doi:10.1016/j.ijpddr.2015.04.002

22. Matovu E, Stewart ML, Geiser F, Brun R, Mäser P, Wallace LJ, Burchmore RJ, Enyaru JC, Barrett MP, Kaminsky R, Seebeck T, de Koning HP (2003) Mechanisms of arsenical and diamidine uptake and resistance in Trypanosoma brucei. Euk Cell 2:1003-1008

23. Geiser F, Luscher A, de Koning HP, Seebeck T, Mäser P (2005) Molecular pharmacology of adenosine transport in Trypanosoma brucei: P1/P2 revisited. Mol Pharmacol 68(3):589-595

24. Bernhard SC, Nerima B, Mäser P, Brun R (2007) Melarsoproland pentamidine-resistant Trypanosoma brucei rhodesiense populations and their cross-resistance. Int $\mathrm{J}$ Parasitol 37(13):1443-1448. doi:10.1016/j.ijpara.2007.05.007

25. Baltz T, Baltz D, Giroud C, Crockett J (1985) Cultivation in a semi-defined medium of animal infective forms of Trypanosoma brucei, T. equiperdum, T. evansi, T. rhodesiense and T. gambiense. EMBO J 4(5):1273-1277

26. Lanham S, Godfrey D (1970) Isolation of salivarian trypanosomes from man and other mammals using DEAE-cellulose. Exp Parasitol 28:521-534

27. Räz B, Iten M, Grether-Bühler Y, Kaminsky R, Brun R (1997) The Alamar Blue assay to determine drug sensitivity of African trypanosomes in vitro. Acta Trop 68:139-147

28. Gould M, Vu X, Seebeck T, de Koning H (2008) Propidium iodide-based methods for monitoring drug action in the kinetoplastidae: comparison with the Alamar Blue assay. Anal Biochem 382:87-93

29. Nilsson D, Gunasekera K, Mani J, Osteras M, Farinelli L, Baerlocher L, Roditi I, Ochsenreiter T (2010) Spliced leader trapping reveals widespread alternative splicing patterns in the highly dynamic transcriptome of Trypanosoma brucei. PLoS Pathog 6(8):e1001037

30. Li H, Ruan J, Durbin R (2008) Mapping short DNA sequencing reads and calling variants using mapping quality scores. Genome Res 18:1851-1858

31. Anders S, Huber W (2010) Differential expression analysis for sequence count data. Genome Biol 11(10):R106. doi:10.1186/gb2010-11-10-r106

32. Chevreux B, Pfisterer T, Drescher B, Driesel A, Müller W, Wetter T, Suhai S (2004) Using the miraEST assembler for reliable and automated mRNA transcript assembly and SNP detection in sequenced ESTs. Genome Res 14:1147-1159

33. Otto T, Dillon G, Degrave W, Berriman M (2011) Rapid annotation transfer tool. Nuc Acids Res 39:e57

34. Swain M, Tsai I, Assefa S, Newbold C, Berriman M, Otto T (2012) A post-assembly genome-improvement toolkit (PAGIT) to obtain annotated genomes from contigs. Nat Protoc 7:1260-1284

35. Giardine B, Riemer C, Hardison R, Burhans R, Elnitski L, Shah P, Zhang Y, Blankenberg D, Albert I, Taylor J, Miller W, Kent W, Nekrutenko A (2005) Galaxy: a platform for interactive largescale genome analysis. Genome Res 15:1451-1455

36. Li H, Handsaker B, Wysoker A, Fennell T, Ruan J, Homer N, Marth G, Abecasis G, Durbin R, Subgroup GPDP (2009) The sequence alignment/map format and SAMtools. Bioinforma Oxf Engl 25:2078-2079

37. Carver T, Berriman M, Tivey A, Patel C, Böhme U, Barrell B, Parkhill J, Rajandream M (2008) Artemis and ACT: viewing, annotating and comparing sequences stored in a relational database. Bioinforma Oxf Engl 24:2672-2676

38. Biebinger S, Wirtz L, Lorenz P, Clayton C (1997) Vectors for inducible expression of toxic gene products in bloodstream and procyclic Trypanosoma brucei. Mol Biochem Parasitol 85:99-112

39. Burkard G, Fragoso C, Roditi I (2007) Highly efficient stable transformation of bloodstream forms of Trypanosoma brucei. Mol Biochem Parasitol 153(2):220-223

40. Hirumi H, Hirumi K (1989) Continuous cultivation of Trypanosoma brucei blood stream forms in a medium containing a low concentration of serum protein without feeder cell layers. J Parasitol 75(6):985-989

41. Alsford S, Horn D (2008) Single-locus targeting constructs for reliable regulated RNAi and transgene expression in Trypanosoma brucei. Mol Biochem Parasitol 161(1):76-79. doi:10. 1016/j.molbiopara.2008.05.006

42. Alsford S, Kawahara T, Glover L, Horn D (2005) Tagging a $T$. brucei RRNA locus improves stable transfection efficiency and circumvents inducible expression position effects. Mol Biochem Parasitol 144(2):142-148. doi:10.1016/j.molbiopara.2005.08.009

43. Schumann Burkard G, Jutzi P, Roditi I (2011) Genome-wide RNAi screens in bloodstream form trypanosomes identify drug transporters. Mol Biochem Parasitol 175(1):91-94. doi:10.1016/j. molbiopara.2010.09.002

44. Lüscher A, Lamprea-Burgunder E, Graf FE, de Koning HP, Mäser P (2014) Trypanosoma brucei adenine-phosphoribosyltransferases mediate adenine salvage and aminopurinol susceptibility but not adenine toxicity. Int J Parasitol Drugs Drug Resist 4(1):55-63. doi:10.1016/j.ijpddr.2013.12.001

45. Vincent IM, Creek D, Watson DG, Kamleh MA, Woods DJ, Wong PE, Burchmore RJ, Barrett MP (2010) A molecular mechanism for eflornithine resistance in African trypanosomes. PLoS Pathog 6(11):e1001204. doi:10.1371/journal.ppat.1001204

46. Walder JA, Eder PS, Engman DM, Brentano ST, Walder RY, Knutzon DS, Dorfman DM, Donelson JE (1986) The 35-nucleotide spliced leader sequence is common to all trypanosome messenger RNA's. Science 233(4763):569-571

47. Alibu VP, Richter C, Voncken F, Marti G, Shahi S, Renggli CK, Seebeck T, Brun R, Clayton C (2006) The role of Trypanosoma brucei MRPA in melarsoprol susceptibility. Mol Biochem Parasitol 146(1):38-44

48. Lüscher A, Nerima B, Mäser P (2006) Combined contribution of TbAT1 and TbMRPA to drug resistance in Trypanosoma brucei. Mol Biochem Parasitol 150(2):364-366

49. Stewart ML, Burchmore RJ, Clucas C, Hertz-Fowler C, Brooks K, Tait A, Macleod A, Turner CM, De Koning HP, Wong PE, Barrett MP (2010) Multiple genetic mechanisms lead to loss of 
functional TbAT1 expression in drug-resistant trypanosomes. Eukaryot Cell 9(2):336-343. doi:10.1128/EC.00200-09

50. Vanhamme L, Renauld H, Lecordier L, Poelvoorde P, Den Van, Abbeele J, Pays E (2004) The Trypanosoma brucei reference strain TREU927/4 contains T. brucei rhodesiense-specific SRA sequences, but displays a distinct phenotype of relative resistance to human serum. Mol Biochem Parasitol 135:39-47

51. Vanhamme L, Paturiaux-Hanocq F, Poelvoorde P, Nolan DP, Lins L, Van Den Abbeele J, Pays A, Tebabi P, Van Xong H, Jacquet A, Moguilevsky N, Dieu M, Kane JP, De Baetselier P, Brasseur R, Pays E (2003) Apolipoprotein L-I is the trypanosome lytic factor of human serum. Nature 422(6927):83-87. doi:10. 1038/nature01461

52. Munday JC, Tagoe DN, Eze AA, Krezdorn JA, Rojas Lopez KE, Alkhaldi AA, McDonald F, Still J, Alzahrani KJ, Settimo L, De Koning HP (2015) Functional analysis of drug resistance-associated mutations in the Trypanosoma brucei adenosine transporter 1 (TbAT1) and the proposal of a structural model for the protein. Mol Microbiol 96(4):887-900. doi:10.1111/mmi. 12979

53. Munday JC, Settimo L, de Koning HP (2015) Transport proteins determine drug sensitivity and resistance in a protozoan parasite, Trypanosoma brucei. Front Pharmacol 6:32. doi:10.3389/fphar. 2015.00032

54. Volpon L, Do I, Young C, Frasch A, Gehring K (2005) NMR structural study of TcUBP1, a single RRM domain protein from Trypanosoma cruzi: contribution of a beta hairpin to RNA binding. Biochemistry 44(10):3708-3717
55. Hartmann C, Benz C, Brems S, Ellis L, Luu V, Stewart M, D’Orso I, Busold C, Fellenberg K, Frasch AC, Carrington M, Hoheisel J, Claytin C (2007) Small trypanosome RNA-binding proteins TbUBP1 and TbUBP2 influence expression of F-box protein mRNAs in bloodstream trypanosomes. Euk Cell 6(11):1964-1978

56. Pyana P, Van Reet N, Mumba Ngoyi D, Ngay Lukusa I, Karhemere Bin Shamamba S, Buscher P (2014) Melarsoprol sensitivity profile of Trypanosoma brucei gambiense isolates from cured and relapsed sleeping sickness patients from the Democratic Republic of the Congo. PLoS Negl Trop Dis 8(10):e3212. doi:10.1371/journal.pntd.0003212

57. Cassola A, Frasch AC (2009) An RNA recognition motif mediates the nucleocytoplasmic transport of a trypanosome RNAbinding protein. J Biol Chem 284(50):35015-35028

58. Alsford S, Eckert S, Baker N, Glover L, Sanchez-Flores A, Leung KF, Turner DJ, Field MC, Berriman M, Horn D (2012) Highthroughput decoding of antitrypanosomal drug efficacy and resistance. Nature 482(7384):232-236. doi:10.1038/nature10771

59. Carver T, Böhme U, Otto T, Parkhill J, Berriman M (2010) BamView: viewing mapped read alignment data in the context of the reference sequence. Bioinformatics 26:676-677

60. Krumsiek J, Arnold R, Rattei T (2007) Gepard: a rapid and sensitive tool for creating dotplots on genome scale. Bioinforma Oxf Engl 23:1026-1028

61. Arnold K, Bondoli L, Kopp J, Schwede T (2006) The SWISSMODEL workspace: a web-based environment for protein structure homology modelling. Bioinformatics 22(2):195-201 